INTERNATIONAL JOURNAL OF

SYSTEMATIC BACTERIOLOGY

Vol. 17, No. 4 October 1967 pp. 323-341

Copyright 1967, Jowa State University Press

\title{
AMENDED DESCRIPTION OF RAMIBACTERIUM ALACTOLYTICUM PRÉVOT AND TAFFANEL WITH PROPOSAL OF A NEOTYPE STRAIN ${ }^{1}$
}

Lillian V. Holdeman, Elizabeth P. Cato and W.E. C. Moore

Anaerobe Laboratory, Department of Veterinary Science

Research Division, Virginia Polytechnic Institute Blacksburg, Virginia 24061

ABSTRACT. Prévot's strain DO-4 (VPI strain $0416)$ is proposed as the neotype strain of Ramibacterium alactolyticum Prévot and Taf fanel (1942). This strain represents members of the genus Ramibacterium formerly assigned to R. alactolyticum, R. pleuriticum, and $\underline{R}$. dentium, which are objective synonyms. alactolyticum is an aerobic, Gram-positive, catalase-negative, pleomorphic rod that produces alcohols and acetic, butyric, caprioc, and often caprylic acids fromglucose, apparently by condensation of $2-c a r b o n$ intermediates with carboxylic acids in the medium. The organism is morphologically similar to the anaerobic corynebacteria, but can be differentiated from catalase-negative anaerobic corynebacteria by fermentation products and by several other properties as described in this study. R. alactolyticum has been isolated from human infections in Europe and in the United States.

Ramibacte rium alactolyticum Prévot and Taffanel (1942), R. pleuriticum Prévot, Raynaud, and Digeon (1947), and $\underline{R}$. dentium Vinzent and Reynes (1947) were described as species of anaerobic, pleomorphic, non-sporeforming, nonmotile, Gram-positive rods differing from each other pri-

1 This research was supported in part by the Division of Systematic Biology of the National Science Foundation and by the Institute of General Medical Sciences of the National Institutes of Health. 
marily in their ability to produce acetylmethylcarbinol, indol, and hydrogen sulfide and in the products detected from their fermentation of glucose. In order to characterize further these species and to identify with certainty some of our own cultures, labeled cultures of these specics were obtained for study.

\section{CULTURES}

Thirty-thrce strains are included in this study. Through the courtesy of Dr. A.R. Prévot of the Pasteur Institute, Paris, all of the labeled strains of the species of $\underline{R}$. alactolyticum, R. pleuriticum, and $\underline{R}$. dentium now in his collection we re obtained. These include one cotype strain of $\underline{R}$. alactolyticum, 23 cotype strains of $\underline{R}$. pleuriticum, and 3 strains which Dr. Prévot had labeled $\underline{\text { R. dentium. To our }}$ knowledge, none of the strains from which the original descriptions of these 3 species were derived is extant. Four previously unidentified strains, isolated in the United States, are included in this study. Two of these were cultures isolated from pleural infections and referred to one of us by the California State Health Department Laboratory, and one was isolated from a pleural infection and referred to one of us by the Arkansas State Health Laboratory. One strain, which was the only organism isolated from the infected stump of an amputated leg, was sent to us by Dr. Elizabeth Petran. Maryland State Health Laboratory. Also included in this study are two strains, received labeled as species of Corynebacterium, which have characteristics similar to the other strains of this group.

\section{METHODS}

Cultures were grown using the anaerobic techniques described by Moore (1966), and characteristics of the organisms were determined by the methods described by Moore and Cato (1965), and Moore, Cato, and Holdeman (1966), with additions or exceptions as noted below.

Peptone-yeast extract medium: The basal medium, peptone-yeast extract (PY), cuntained 2 g peptone and $1 \mathrm{~g}$ yeast extract in $100 \mathrm{ml}$ water with L-cysteine hydrochloride, salts, and resazurin as described previously (Moore and Cato 1965).

Indol production: Ehrlich's reagent, added to xylene extracts of 3-week-old cultures in tryptone yeast and in chopped meat (beef) media, was used to test for presence of indol. 
Acetylmethylcarbinol production: Coblentz reagent was used to test for presence of acetylmethylcarbinol in cultures incubated for 3 days in PY-glucose broth. Similarly incubated cultures of Aerobacter aerogenes were used as positive controls for this test.

Hydrogen sulfide production: Cultures in a medium containing $0.05 \mathrm{~g} \mathrm{Na}_{2} \mathrm{~S}_{2} \mathrm{O}_{3} .5 \mathrm{H}_{2} \mathrm{O}, 0.02 \mathrm{~g} \mathrm{FeSO}_{4} .7 \mathrm{H}_{2} \mathrm{O}, 0.5 \mathrm{~g}$ glucose, 1 g yeast extract, $2 \mathrm{~g}$ peptone, $0.5 \mathrm{~g} \mathrm{NaCl}, 0.05 \mathrm{~g} \mathrm{L-}$ cysteine hydrochloride, and resazurin in $100 \mathrm{ml}$ water were observed for 3 weeks for development of a black color, which indicated $\mathrm{H}_{2} \mathrm{~S}$ production.

Nitrate reduction: Three-week-old growth in BBL indolnitrate medium was used to test for reduction of nitrate.

Starch hydrolysis: Three-week-old cultures in a medium containing $1 \%$ soluble starch were tested with Gram's iodine for indication of starch hydrolysis. Immediate development of a dark purple color when a few drops of iodine solution were added to the broth culture indicated that starch was still present in the medium and was not entirely hydrolyzed; absence of dark purple color indicated complete hydrolysis.

Esculin hydrolysis: To detect hydrolysis of esculine, a few drops of $1 \%$ aqueous ferric ammonium citrate were added to a 3-week-old culture in the esculin medium. Development of a black colloid indicated that the esculin had been hydrolyzed. To assure that the black color was not caused by reaction with $\mathrm{H}_{2} \mathrm{~S}$, ferric ammonium citrate also was added to a 3-week-old culture in another medium (usually fructose) showing comparable growth.

Catalase: Sensitivity of the catalase test was increased by exposing surface growth on pre-reduced anaerobically sterilized agar slants to atmospheric oxygen for 30 minutes before adding the $3 \%$ hydrogen peroxide. It was found in this laboratory that some organisms known to be catalase positive failed to produce detectable catalase under highly reduced conditions and were catalase positive only after growth on reduced medium was exposed to atmospheric oxygen.

Reduction of neutral red: At the time of inoculation, two drops (about $0.03 \mathrm{ml}$ ) of autoclaved neut ral red solution $(0.1$ $\mathrm{g}$ in $100 \mathrm{ml} 60 \%$ ethanol) were added aseptically to tubes containing $4 \mathrm{ml}$ of $\mathrm{PY}-\mathrm{f}$ ructose medium. Cultures were examined after incubation for one or more days. Presence of red color indicated that the neutral red had not been reduced; absence of color indicated that the neutral red had been reduced. Uninoculated tubes remained red.

Reduction of resazurin: Tubes of molten PY-glucose agar 
deeps were inoculated and loosely covered with sterile aluminum foil to allow penetration of oxygen into the top layers of the modium and oxidation of the resazurin in this layer. Reduction of rcsazurin was indicated by the subsequent disappearance of the red color.

Motility: Young cultures ( 6 to 16 hours) in chopped meat medium were examined microscopically for motile cells by placing a drop of culture on a slide and immediately looking for motile cells $(490 x)$. Wet-mount preparations under cover clips also were sometimes used.

Threonine medium: Conversion of threonine to propionate was tested for by gas chromatography of cultures grown in a medium containing $0.5 \mathrm{~g}$ yeast extract, $0.5 \mathrm{~g}$ tryptone, 0.2 g K $\mathrm{K}_{2} \mathrm{HOO}_{4}, 0.05 \mathrm{~g}$ glucose, $0.3 \mathrm{~g}$ DL-threonine, resazurin, salts, and L-cysteine hydrochloride in $100 \mathrm{ml}$ of distilled water. The amount of propionate produced in the threonine medium was compared with that produced in a threoninefree medium to delect a significant increase in propionate production.

Conversion and utilization of pyruvate or lactate: Substrate was "converted" when the increase in products detected (compared with PY) accounted for the disappearance of the substrate; substrate was considered to be "utilized" when products detected did not account for the disappearance of the substrate.

Growth in bile medium: The bile medium contained $2 \mathrm{~g}$ oxgall and $0.1 \mathrm{~g}$ sodium deoxycholate in $100 \mathrm{ml}$ PY-glucose broth. The amount of growth was compared with growth in PY-glucose broth to determine growth inhibition or stimulation.

Growth on solid media: Characteristics of surface colonics were described from surface-streaked roll tubes and from horse blood agar $(4 \mathrm{ml}$ blood in $100 \mathrm{ml}$ brain heart infusion agar) and egg yolk agar plates. The plates were incubated for 2 days in anaerobic jars with catalysts in an atmosphere of $97 \%$ hydrogen and $3 \%$ carbon dioxide. Growth on egg yolk agar was observed for, 1) development of an opaque zone of precipitate in the agar around the colonies (lecithinase reaction), 2) development of an iridescent "pear$y^{\prime \prime}$ luster over and around the colonies and an opaque zone in the agar under the pearly layer (lipasc reaction), and 3) clearing of the agar around the colony or zone of reaction (lytic action).

Modified McClung-Toabe egg yolk agar: Mix 20 g peptone

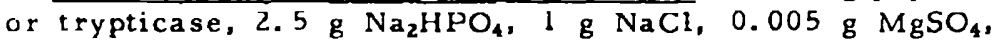


$1 \mathrm{~g}$ glucose and $12.5 \mathrm{~g}$ agar in $500 \mathrm{ml}$ distilled water. Adjust $\mathrm{pH}$ to 7.3 to 7.4 , autoclave at $121^{\circ} \mathrm{C}$ for 15 minutes, and cool to $52^{\circ} \mathrm{C}$. Soak egg in $70 \%$ alcohol for about 30 minutes. Separate yolk from white, add yolk to cooled medium, mix, and pour plates. Use plates immediately or store in an anae robic atmos phere.

Analysis of data: Results obtained on each of these cultures were coded, the characteristics of each strain were compared with the characteristics of every other strain, and the characteristics of the average strain were determined. The data also were analyzed for subgroups by Dr. Rita Colwell, Georgetown University.

\section{RESULTS AND DISCUSSION}

Results of our analyses of the 33 strains examined, as well as reactions for $\underline{R}$. alactolyticum, $R$. pleuriticum, and $\underline{R}$. dentium published in the original descriptions of the species, are given in Table 1. The results of 55 sets of analyses on the 33 strains included in the study are summarized in the first column. Characteristics of $\underline{R}$. alactolyticum, Prévot DO-4 (VPI 0416), are given in column 2 of Table 1 . This strain is a cotype strain and, to our knowledge, the only available labeled strain of $R$. alactolyticum. Results of 29 sets of analyses of 23 cotype strains of $\underline{R}$. pleuriticum and results of nine sets of analyses on the only three available labeled strains of $\underline{R}$. dentium are given in columns 3 and 4 respectively. Results obtained on the four strains isolated from human clinical material in the United States are presented in column 5 .

Characteristics previously described to differentiate among $\underline{R}$. alactolyticum, $\underline{R}$. pleuriticum, and $\underline{R}$. dentium are shown in Table 2. Although all labeled strains studied were similar, none of them had characteristics exactly corresponding to the differential reactions given for any species in Table 2. The results observed on cultures received from Dr. Prévot were in general agreement with those he had found, some of which also differed slightly from the published descriptions. All 33 strains reduced neutral red and none produced acetylmethylcarbinol or indol, although appropriate positive and negative reactions were obtained with other species of non-sporeforming anaerobic bacteria in these three tests. Only one of the 33 strains tested produced detectable $\mathrm{H}_{2} \mathrm{~S}$ in our medium. The test used for $\mathrm{H}_{2} \mathrm{~S}$, however, is not so sensitive as some others which may have been used. 
TABLE 1. CHARACTERISTICS OF STRAINS OF RAMIBACTERIA

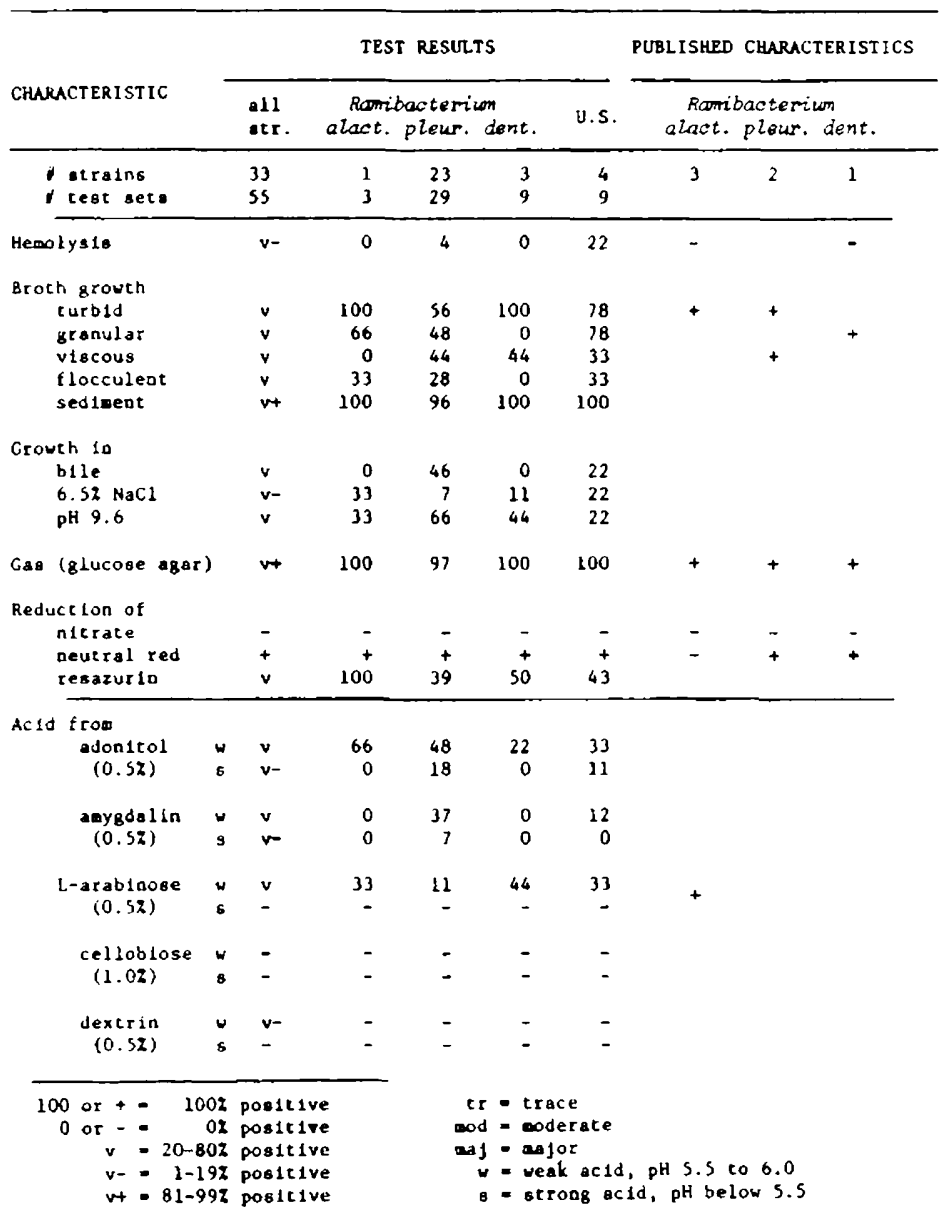


TABLE 1 (cont. $)^{2}$

\begin{tabular}{|c|c|c|c|c|c|c|c|c|c|}
\hline \multirow[b]{2}{*}{ CHARACTERISTIC } & \multicolumn{6}{|c|}{ TEST RESURTS } & \multirow{2}{*}{$\frac{\text { PUBLISHED }}{\begin{array}{r}\text { Ramib } \\
\text { alact. }\end{array}}$} & \multirow{2}{*}{\multicolumn{2}{|c|}{$\begin{array}{l}\text { CHARACTERISTICS } \\
\text { bacterium } \\
\text { plewr. dent. }\end{array}$}} \\
\hline & & $\begin{array}{l}\text { al1 } \\
\text { str. }\end{array}$ & $\begin{array}{l}\text { Romi } \\
\text { alact. }\end{array}$ & $\begin{array}{l}\text { pacteri } \\
\text { pleur. }\end{array}$ & dent. & U.s. & & & \\
\hline $\begin{array}{c}\text { dulcttol } \\
(1.0 z)\end{array}$ & $\begin{array}{l}w \\
a\end{array}$ & - & - & - & - & - & & & \\
\hline $\begin{array}{c}\text { DL-erythritol } \\
(0.52)\end{array}$ & w & $\begin{array}{l}v \\
v-\end{array}$ & $\begin{array}{l}0 \\
0\end{array}$ & $\begin{array}{r}43 \\
4\end{array}$ & $\begin{array}{l}0 \\
0\end{array}$ & $\begin{array}{r}25 \\
0\end{array}$ & & & \\
\hline $\begin{array}{r}\text { esculin } \\
(0.5 \%)\end{array}$ & $\begin{array}{l}w \\
8\end{array}$ & $\begin{array}{l}v- \\
v-\end{array}$ & $\begin{array}{l}0 \\
0\end{array}$ & $\begin{array}{r}32 \\
7\end{array}$ & $\begin{array}{l}0 \\
0\end{array}$ & $\begin{array}{r}12 \\
0\end{array}$ & & & \\
\hline $\begin{array}{c}D-f \text { ructooe } \\
(1.07)\end{array}$ & w & $\begin{array}{l}w+ \\
w+\end{array}$ & $\begin{array}{l}100 \\
100\end{array}$ & $\begin{array}{l}97 \\
90\end{array}$ & $\begin{array}{l}89 \\
89\end{array}$ & $\begin{array}{l}89 \\
78\end{array}$ & + & & + \\
\hline $\begin{array}{c}D-8 \text { alactose } \\
(1.0 x)\end{array}$ & $w$ & $\begin{array}{l}v- \\
v-\end{array}$ & $\begin{array}{l}0 \\
0\end{array}$ & $\begin{array}{l}7 \\
0\end{array}$ & $\begin{array}{r}22 \\
0\end{array}$ & $\begin{array}{l}11 \\
11\end{array}$ & + & + & \\
\hline $\begin{array}{c}\text { glucose } \\
(1.02) .\end{array}$ & w & $\begin{array}{l}v+ \\
v\end{array}$ & $\begin{array}{l}100 \\
100\end{array}$ & $\begin{array}{l}93 \\
52\end{array}$ & $\begin{array}{l}100 \\
100\end{array}$ & $\begin{array}{r}100 \\
78\end{array}$ & + & + & + \\
\hline $\begin{array}{l}\text { glycerol } \\
(1.0 x)\end{array}$ & w & - & - & - & - & - & & & - \\
\hline $\begin{array}{c}\text { glycogen } \\
(0.5 x)\end{array}$ & $\begin{array}{l}w \\
\text { s }\end{array}$ & $\overline{-}$ & - & - & - & - & & & \\
\hline $\begin{array}{c}\text { Inositol } \\
(1.0 z)\end{array}$ & w & - & - & $\overline{-}$ & - & - & & & \\
\hline $\begin{array}{c}\text { 1nulin } \\
(1.0 \%)\end{array}$ & s & - & - & - & - & - & & & \\
\hline $\begin{array}{r}\text { lactose } \\
(1.0 z)\end{array}$ & $\begin{array}{l}\text { w } \\
\text { s }\end{array}$ & $\begin{array}{l}\mathrm{v}- \\
-\end{array}$ & $\begin{array}{l}0 \\
-\end{array}$ & $\begin{array}{l}4 \\
-\end{array}$ & $\begin{array}{l}0 \\
-\end{array}$ & $\because$ & - & - & - \\
\hline $\begin{array}{r}D(+)-\text { thal tose } \\
(1.0 z)\end{array}$ & : & $\begin{array}{l}v- \\
v-\end{array}$ & $\begin{array}{l}0 \\
0\end{array}$ & $\begin{array}{l}8 \\
4\end{array}$ & $\begin{array}{l}0 \\
0\end{array}$ & $\begin{array}{l}0 \\
0\end{array}$ & & 50 & - \\
\hline $\begin{array}{c}\text { D-mannitol } \\
(1.0 \%)\end{array}$ & $w$ & $v+$ & $\begin{array}{l}66 \\
33\end{array}$ & $\begin{array}{l}82 \\
54\end{array}$ & $\begin{array}{l}67 \\
22\end{array}$ & $\begin{array}{l}86 \\
43\end{array}$ & & & + \\
\hline $\begin{array}{r}D(+)-\text { mannose } \\
(1.0 z)\end{array}$ & 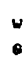 & $\begin{array}{l}v- \\
-\end{array}$ & $\frac{0}{-}$ & $\begin{array}{l}0 \\
-\end{array}$ & 11 & 11 & & & \\
\hline $\begin{array}{l}\text { melez1tose } \\
(0.5 z)\end{array}$ & : & $\begin{array}{l}v \\
-\end{array}$ & $\begin{array}{l}0 \\
-\end{array}$ & 39 & $\begin{array}{l}0 \\
-\end{array}$ & 11 & & & \\
\hline
\end{tabular}


TABLE 1 (Cont.) ${ }^{3}$

\begin{tabular}{|c|c|c|c|c|c|c|c|c|c|}
\hline \multirow[b]{2}{*}{ CHARACTERISTIC } & & \multicolumn{5}{|c|}{ TEST RESULTS } & \multicolumn{3}{|c|}{ PUBLISHED CHARACTERISTICS } \\
\hline & & $\begin{array}{l}\text { all } \\
\text { str. }\end{array}$ & $\begin{array}{l}\text { Romi } \\
\text { alact. }\end{array}$ & $\begin{array}{l}\text { bacter } \\
\text { pieur. }\end{array}$ & $\begin{array}{l}\text { iion } \\
\text { dent. }\end{array}$ & U.S. & $\begin{array}{l}\text { Romit } \\
\text { alact. }\end{array}$ & $\begin{array}{l}\text { bacteriur } \\
\text { pleur. }\end{array}$ & dent. \\
\hline $\begin{array}{l}\text { Del1blose } \\
(0.5 x)\end{array}$ & $\dot{v}$ & v- & - & - & - & - & & & \\
\hline $\begin{array}{l}\text { pectin } \\
(1.02)\end{array}$ & 5 & $\begin{array}{l}v \\
v-\end{array}$ & $\begin{array}{r}200 \\
33\end{array}$ & $\begin{array}{r}31 \\
3\end{array}$ & $\begin{array}{l}78 \\
33\end{array}$ & $\begin{array}{r}67 \\
0\end{array}$ & & & \\
\hline $\begin{array}{l}\text { raf finose } \\
(1.0 x)\end{array}$ & $\begin{array}{l}w \\
\text { s }\end{array}$ & $\begin{array}{l}v- \\
-\end{array}$ & $\begin{array}{l}0 \\
-\end{array}$ & $\begin{array}{l}0 \\
-\end{array}$ & $\begin{array}{r}12 \\
-\end{array}$ & $\begin{array}{l}0 \\
-\end{array}$ & & & \\
\hline $\begin{array}{l}\text { rhamnose } \\
(1.0 z)\end{array}$ & s & v- & - & $\begin{array}{l}? \\
-\end{array}$ & 11 & $\begin{array}{l}0 \\
-\end{array}$ & & & \\
\hline $\begin{array}{r}D(-)-\text { ribose } \\
(0.5 \pi)\end{array}$ & 5 & $\begin{array}{l}v \\
v-\end{array}$ & $\begin{array}{r}66 \\
0\end{array}$ & $\begin{array}{r}48 \\
0\end{array}$ & $\begin{array}{l}56 \\
22\end{array}$ & $\begin{array}{l}44 \\
22\end{array}$ & & & \\
\hline $\begin{array}{l}\text { salicin } \\
(1.0 z)\end{array}$ & w & - & - & $\overline{-}$ & - & $\overline{-}$ & & & \\
\hline $\begin{array}{c}D-\text { sorbitol } \\
(1.07)\end{array}$ & $\begin{array}{l}w \\
s\end{array}$ & $\begin{array}{l}v \\
v-\end{array}$ & $\begin{array}{l}0 \\
0\end{array}$ & $\begin{array}{r}14 \\
7\end{array}$ & $\begin{array}{l}0 \\
0\end{array}$ & $\begin{array}{l}50 \\
38\end{array}$ & & & - \\
\hline $\begin{array}{r}\text { L-sorbose } \\
(1.02)\end{array}$ & w & $\begin{array}{l}\text { v- } \\
\text { - }\end{array}$ & $\begin{array}{l}0 \\
-\end{array}$ & $\begin{array}{l}3 \\
-\end{array}$ & 22 & 22 & & & \\
\hline $\begin{array}{c}\text { starch } \\
(1.0 z)\end{array}$ & 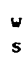 & $\begin{array}{l}- \\
-\end{array}$ & - & - & - & - & & so & - \\
\hline $\begin{array}{c}\text { sucrose } \\
(1.0 z)\end{array}$ & $\dot{s}$ & v- & $\begin{array}{l}0 \\
-\end{array}$ & $\begin{array}{l}0 \\
-\end{array}$ & $\begin{array}{l}0 \\
-\end{array}$ & 11 & & 50 & \\
\hline $\begin{array}{l}\text { crehalose } \\
(0.52)\end{array}$ & $w$ & $\begin{array}{l}- \\
-\end{array}$ & - & - & - & - & & & \\
\hline $\begin{array}{r}\text { D-xylose } \\
(1.0 z)\end{array}$ & s & $\begin{array}{l}v \\
-\end{array}$ & 100 & 32 & 67 & 78 & + & & \\
\hline
\end{tabular}


TABLE $\&$ (Cont) 4

\begin{tabular}{|c|c|c|c|c|c|c|c|c|}
\hline \multirow[b]{2}{*}{ CHARACTERISTIC } & \multicolumn{5}{|c|}{ TEST RESULTS } & \multirow{2}{*}{$\begin{array}{l}\text { PUBLISHED } \\
\text { Romi } \\
\text { alact. }\end{array}$} & \multicolumn{2}{|c|}{ CHARACTERISTICS } \\
\hline & $\begin{array}{l}\text { all } \\
\text { Btr. }\end{array}$ & $\begin{array}{l}\text { Rami } \\
\text { alaot. }\end{array}$ & $\begin{array}{l}\text { acter } \\
\text { pleur. }\end{array}$ & lent. & U.S. & & $\begin{array}{l}\text { bacteriuo } \\
\text { pleur. }\end{array}$ & dent. \\
\hline \multicolumn{9}{|l|}{ Lactate } \\
\hline converston & vt & 33 & 86 & 88 & 78 & & & \\
\hline ut1l1zation & vt & 66 & 86 & 75 & 78 & & & \\
\hline \multicolumn{9}{|l|}{ Pyruvate } \\
\hline conversion & $v+$ & 100 & 82 & 78 & 67 & & & \\
\hline ut1112at1on & $\mathbf{v}$ & 0 & 34 & 22 & 22 & & & \\
\hline \multicolumn{9}{|l|}{ Threonine (DL) } \\
\hline conversion & - & - & - & - & - & & & \\
\hline \multicolumn{9}{|l|}{ M1lk } \\
\hline curd & $v-$ & 66 & 10 & 0 & 0 & - & - & - \\
\hline digestion & $v-$ & 0 & 7 & 0 & 0 & & - & \\
\hline Gelatin digestion & $v-$ & 0 & 14 & 0 & 11 & - & - & - \\
\hline Chopped beef $d 1_{g}$. & - & - & - & - & - & & & \\
\hline Production of $\mathrm{NH}_{3}$ & & & & & & + & tr & + \\
\hline arginine & $v-$ & 0 & 4 & 33 & 0 & & & \\
\hline threonine & - & - & - & - & - & & & \\
\hline chopped beef & $v-$ & 0 & 7 & 0 & 0 & & & \\
\hline \multicolumn{9}{|l|}{ Hydrolysis of } \\
\hline esculin & - & - & - & - & - & & & \\
\hline 8tarch & - & - & - & - & - & & & \\
\hline hippurate & v- & 0 & 3 & 0 & 0 & & & \\
\hline \multicolumn{9}{|l|}{ Production of } \\
\hline $\begin{array}{l}\text { ladol } \\
\text { skatol }\end{array}$ & - & - & - & - & - & tr & - & $\begin{array}{l}+ \\
-\end{array}$ \\
\hline catalage & - & - & - & - & - & $\star$ & & \\
\hline acetoin (NHC) & - & - & - & - & - & - & - & + \\
\hline $\mathrm{H}_{2} \mathrm{~S}$ & $v=$ & 0 & 4 & 0 & 0 & $\operatorname{tr}$ & So & - \\
\hline
\end{tabular}


TABLE. 1 (Cont. $)^{5}$

\begin{tabular}{|c|c|c|c|c|c|c|c|c|c|}
\hline \multirow[b]{2}{*}{ CHARACTERISTIC } & & \multicolumn{5}{|c|}{ TEST RESULTS } & PUBLISHED $C$ & \multicolumn{2}{|c|}{ CHARACTERISTICS } \\
\hline & & $\begin{array}{l}\text { all } \\
\text { str. }\end{array}$ & $\begin{array}{r}\text { Romi } \\
\text { alact. }\end{array}$ & $\begin{array}{l}\text { acterix } \\
\text { pleur. }\end{array}$ & dent. & U.S. & $\begin{array}{l}\text { Pacmit } \\
\text { alact. }\end{array}$ & $\begin{array}{c}\text { bacteri } \\
\text { pleur. }\end{array}$ & $\pi$ \\
\hline \multicolumn{10}{|c|}{ Acids from glucose } \\
\hline \multirow[t]{3}{*}{ acetic } & tr & + & + & + & + & + & + & + & - \\
\hline & mod & $v$ & 100 & 62 & 33 & 89 & + & + & - \\
\hline & maj & $v-$ & 66 & 3 & 0 & 11 & + & & - \\
\hline \multirow[t]{3}{*}{ proplonic } & $t r$ & $v$ & 0 & 34 & 11 & 33 & & & \\
\hline & mod & - & - & - & - & - & & & \\
\hline & maj & - & - & - & - & - & & & \\
\hline \multirow[t]{3}{*}{ 1sobutyric } & $t r$ & $v-$ & 0 & 3 & 0 & 11 & & & \\
\hline & mod & - & - & - & - & - & & & \\
\hline & $\operatorname{maj}$ & - & - & - & - & - & & & \\
\hline \multirow[t]{3}{*}{ butyric } & $t \mathbf{r}$ & + & + & + & + & + & + & & + \\
\hline & $\bmod$ & vt & 100 & 93 & 89 & 100 & & & \\
\hline & maj & $v-$ & 33 & 0 & 0 & 0 & & & \\
\hline \multirow[t]{3}{*}{ Isovaleric } & cr & - & - & - & - & - & & & \\
\hline & $\bmod$ & - & - & - & - & - & & & \\
\hline & maj & - & - & - & - & - & & & \\
\hline \multirow[t]{3}{*}{ valeric } & cr & $v-$ & 0 & 10 & 0 & 0 & & + & + \\
\hline & mod & - & - & - & - & - & & + & + \\
\hline & mas & - & - & - & - & - & & + & \\
\hline \multirow[t]{3}{*}{ isocaproic } & $t r$ & $v-$ & 0 & 3 & 0 & 11 & & & \\
\hline & $\bmod$ & $v-$ & 0 & 3 & 0 & 0 & & & \\
\hline & $m \mathbf{a j}$ & - & - & - & - & - & & & \\
\hline \multirow[t]{3}{*}{ caproic } & $t r$ & + & + & + & + & + & & & $+(?)$ \\
\hline & mod & + & + & + & + & + & & & \\
\hline & maj & $v$ & 100 & 59 & 100 & 67 & & & \\
\hline \multirow[t]{3}{*}{ heptanolc } & $t r$ & $v-$ & 0 & 3 & 0 & 11 & & & \\
\hline & $\bmod$ & - & - & - & - & - & & & \\
\hline & $\mathbf{m a j}$ & - & - & - & - & - & & & \\
\hline \multirow{3}{*}{ caprylic } & $\operatorname{tr}$ & $v$ & 33 & 52 & 100 & 12 & & & \\
\hline & $\bmod$ & $v$ & 0 & 24 & 100 & 12 & & & \\
\hline & maj & v- & 0 & 0 & 14 & 0 & & & \\
\hline
\end{tabular}


IABLE 1 (Cont.) $)^{0}$

\begin{tabular}{|c|c|c|c|c|c|c|c|c|c|}
\hline \multirow[b]{2}{*}{ CHARACTERISTIC } & & \multicolumn{5}{|c|}{ TEST RESULTS } & \multirow{2}{*}{$\begin{array}{r}\text { PUBLISHED } \\
\text { Ramib } \\
\text { alact. }\end{array}$} & \multirow{2}{*}{\multicolumn{2}{|c|}{$\begin{array}{l}\text { CHARACTERIST } \\
\text { baaterilom } \\
\text { pleur. dent. }\end{array}$}} \\
\hline & & \multirow{2}{*}{$\begin{array}{l}\text { all } \\
\text { str. } \\
\begin{array}{l}v+ \\
v \\
v-\end{array}\end{array}$} & \multicolumn{3}{|c|}{$\begin{array}{l}\text { Ramibacterium } \\
\text { alact. pleur. dent. }\end{array}$} & $\frac{\text { U.S. }}{100}$ & & & \\
\hline form $1 c$ & $\begin{array}{l}\text { tr } \\
\text { mod } \\
\text { maj }\end{array}$ & & $\begin{array}{r}100 \\
100 \\
66\end{array}$ & $\begin{array}{l}97 \\
66 \\
14\end{array}$ & $\begin{array}{l}67 \\
44 \\
11\end{array}$ & $\begin{array}{r}100 \\
78 \\
33\end{array}$ & & & $\begin{array}{l}- \\
-\end{array}$ \\
\hline succintc & $\begin{array}{l}t r \\
\bmod \\
\operatorname{maj}\end{array}$ & $\begin{array}{l}v \\
v- \\
v-\end{array}$ & $\begin{array}{r}33 \\
0 \\
0\end{array}$ & $\begin{array}{r}69 \\
10 \\
3\end{array}$ & $\begin{array}{r}44 \\
22 \\
0\end{array}$ & $\begin{array}{r}78 \\
33 \\
0\end{array}$ & & & $\begin{array}{l}- \\
-\end{array}$ \\
\hline lactic & $\begin{array}{l}t r \\
\bmod \\
\operatorname{mad}\end{array}$ & $\begin{array}{l}v \\
-\end{array}$ & $\begin{array}{l}0 \\
- \\
-\end{array}$ & $\begin{array}{r}34 \\
- \\
-\end{array}$ & $\begin{array}{r}44 \\
- \\
-\end{array}$ & $\begin{array}{r}33 \\
- \\
-\end{array}$ & $\begin{array}{l}+ \\
+\end{array}$ & + & $\begin{array}{l}- \\
-\end{array}$ \\
\hline Alcohole frow $\mathrm{g}$ & Iucos & & & & & & - & 50 & - \\
\hline ethanol & $\begin{array}{l}\text { tr } \\
\bmod \\
\operatorname{maj}\end{array}$ & $\begin{array}{l}v- \\
- \\
-\end{array}$ & $\begin{array}{l}0 \\
-\end{array}$ & $\begin{array}{l}0 \\
- \\
-\end{array}$ & $\begin{array}{l}0 \\
- \\
-\end{array}$ & $\begin{array}{r}22 \\
- \\
-\end{array}$ & & & \\
\hline propanol & $\begin{array}{r}\operatorname{tr} \\
\bmod \end{array}$ & $\begin{array}{l}v- \\
v-\end{array}$ & $\begin{array}{r}66 \\
0\end{array}$ & $\begin{array}{l}3 \\
0\end{array}$ & $\begin{array}{l}0 \\
0\end{array}$ & $\begin{array}{l}22 \\
11\end{array}$ & & & \\
\hline butanol & $\begin{array}{l}t r \\
\bmod \\
\operatorname{mat}\end{array}$ & $\begin{array}{l}v \\
v \\
v-\end{array}$ & $\begin{array}{r}100 \\
33 \\
0\end{array}$ & $\begin{array}{r}69 \\
28 \\
7\end{array}$ & $\begin{array}{r}11 \\
0 \\
0\end{array}$ & $\begin{array}{l}89 \\
67 \\
11\end{array}$ & & & \\
\hline $\begin{array}{l}\text { other } \\
\text { alcohols }\end{array}$ & $\begin{array}{r}t r \\
\bmod \\
\operatorname{maj}\end{array}$ & $\begin{array}{l}v+ \\
v \\
v\end{array}$ & $\begin{array}{r}33 \\
0 \\
0\end{array}$ & $\begin{array}{r}100 \\
48 \\
28\end{array}$ & $\begin{array}{r}56 \\
0 \\
0\end{array}$ & $\begin{array}{r}100 \\
89 \\
56\end{array}$ & & & \\
\hline Other products & & & & & & & & & \\
\hline volatile amin & & & & & & & $\operatorname{tr}$ & 50 & - \\
\hline aldehyder & & & & & & & tr & - & - \\
\hline ketones & & & & & & & - & 50 & - \\
\hline phenol & & & & & & & - & & - \\
\hline cresol & & & & & & & - & & - \\
\hline
\end{tabular}


TABLE $\perp$ (Cont. $)^{?}$

Additional characteristics:

Broth cultures, after incubation for 1 to 2 days, contained Gram-positive, pleomorphic rods with rounded and tapered ends occurring singly, in pairs in $V$ and $Y$ arrangements, and in clumps; about half the broth cultures also contained some decolorized cells. Cells ranged in length from 0.8 to $11.0 \mu$ and in diameter from 0.2 to $0.8 \mu$; swellings up to $1.6 \mu$ were seen. The average cell size (minimum to maximum) was 0.4 to $0.5 \mu$ in diameter by 1.9 to $5.6 \mu$ in length.

Temperature for optimum growth was $37^{\circ} \mathrm{C}$, although some strains grew equally well at 30 or $45^{\circ} \mathrm{C}$. Lowest terminal $\mathrm{pH}$ in glucose medium was from 4.9 to 5.9 , usually 5.0 to 5.5. Lowest terminal $\mathrm{pH}$ in glucose was not detected until cultures had been incubated for 7 days and usually occurred after incubation for 2 to 3 weeks. No fetid odor was detected.

Most strains grew in pre-boiled thioglycollate medium and in PRAS (pre-reduced, anaerobically sterilized) nutrient broth but not in aerobic glucose or aerobic lactate broths. No lecithinase, lipase, or lytic reaction was produced on modified McClung-Toabe egg yolk agar. No strain converted threonine to propionate. 


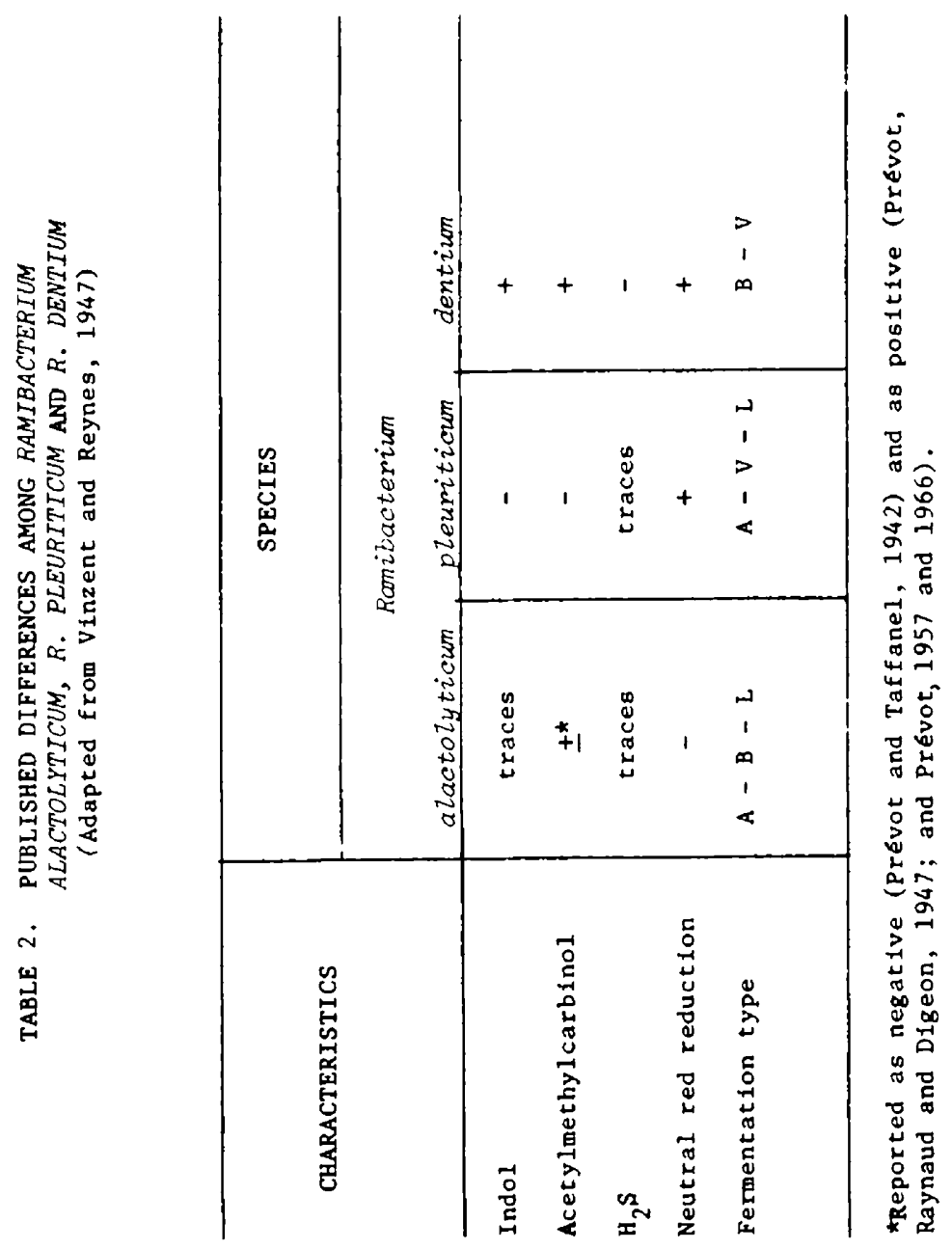


From glucose, all strains produced acetic, butyric, caproic, and often caprylic acids, frequently with corresponding alcohols, formic acid, and trace amounts of propionic acid. We believe that the original descriptions that include valeric or omit butyric and caproic acids probably are in error and resulted from limitations of earlier procedures used for analysis of acid fermentation products. In cases where acid products were determined by chromatography in Dr. Prévot's laboratory, acetic, butyric, and caproic acids were detected.

No well-defined subgroups could be found among the 33 strains studied, either by visual analysis of data or by computer comparisons of each strain against all other strains. Neither could any strain be differentiated as a separate species. Of 274 coded characteristics for each of 55 sets of analyses, therewas an average of 66 positive characteristics per strain in this group. A hypothetical average strain, defined as positive for the most frequently-occurring 66 positive characteristics and negative for the remaining 208, was compared against all 33 strains. Of the four strains most similar to this "average strain," the first strain was labeled $\underline{R}$. dentium; the second, $\underline{R}$. pleuriticum; the third (mislabeled), Corynebacterium diphtheroides; and the fourth was the strain labeled $\underline{\mathrm{R}}$. alactolyticum.

The differences in characteristics amongorganisms previously ascribed to the three species $\underline{R}$. alactolyticum, $\underline{R}$. dentium, and $R$. pleuriticum are not correlated and overall are so slight that these organisms form a single group. We therefore propose Prévot's strain DO-4 (VPI 0416) as the neotype strain to represent the species previously designated as $R$. alactolyticum, R. pleuriticum, and $\underline{R}$. dentium, which names are then objective synonyms. The name of the species, by rule of priority, is $R$. alactolyticum Prévot and Taffanel, 1942. Three strains have been deposited in the American Type Culture Collection: 1) ATCC 23263 (Prévot DO-4, VPI 0416), the proposed neotype strain, isolated from purulent pleurisy; 2) ATCC 19301 (VPI 0573), a culture isolated from a human pleural infection in the United States; and 3) a strain which produces larger amounts of caprylic acid, ATCC 23264 (Prévot 1218, VPI 1691), originally labeled R. pleuriticum and isolated from a lung abscess. 


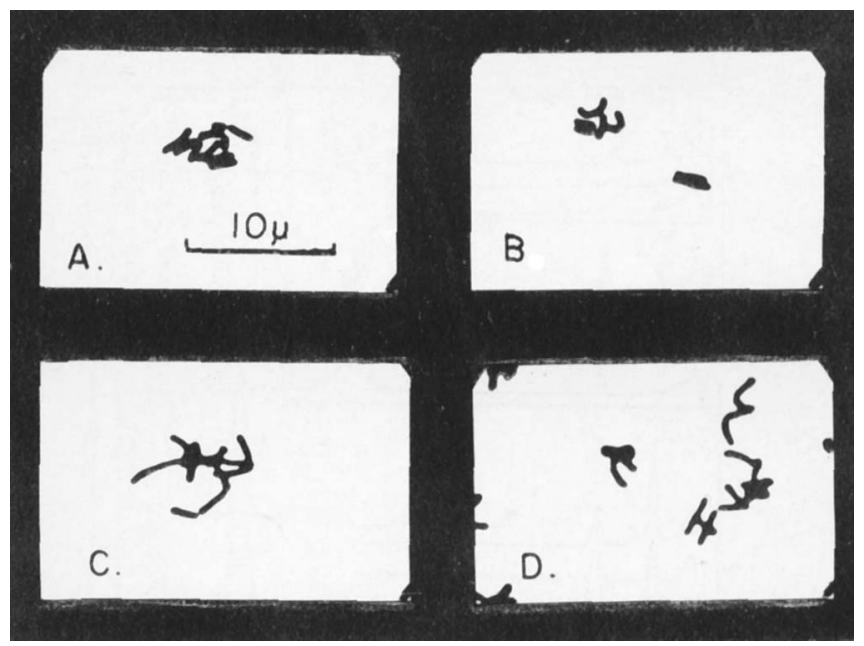

Figure I. Photomicrographs of Gram's stains of 482 hour cultures of $\underline{R}$. alactolyticum, neotype strain; $A$ and $B$ in peptone-yeast extract medium, $C$ and $D$ in peptone-yeast extract-glucose medium.

\section{DISTINCTIVE CHARACTERISTICS OF R. ALACTOLYTICUM}

Ramibacterium alactolyticum is a pleomorphic, Grampositive, anaerobic, catalase-negative, non-sporeforming, nonmotile, fermentative rod. Colonies on agar surface are circular, entire, convex to pulvinate, white opaque or semiopaque, glistening, smooth, pinpoint to $2 \mathrm{~mm}$ in diameter, and may be slightly hemolytic. R. alactolyticum is morphologically similar to the anaerobic corynebacteria and propionibacteria, but usually has a distinctive $V$ arrangement of pairs of slightly curved cells resembling distant flying sea gulls (Fig. l). However, this same cellular arrangement is sometimes seen in stained preparations of the anaerobic corynebacteria. $\underline{R}$. alactolyticum is differentiated with certainty from the anaerobic corynebacteria by major differences in fermentation products from glucose. ‥ alactolyticum produces acetic, butyric, caproic, and usually caprylic acids and often ethanol, butanol, and hexanol. Formic, succinic, or lactic acids and traces of propionic acid and propyl alcohol also are sometimes detected (Fig. 2). Propionibacterium acnes (‥ acnes) produces acetic, propionic, and traces of isovaleric (Fig. 3), variable amounts 

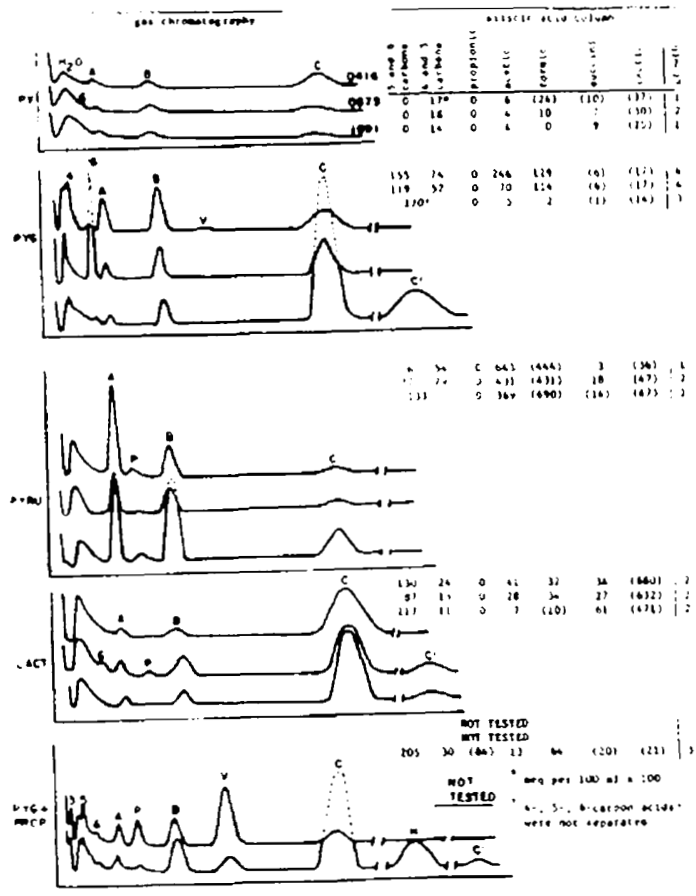

Figure 2. Chromatographic analyses of three strains of R. alactolyticum in 5 media.

Media:

PY = peptone-yeast extract

PYG - PY-glucose

PYRU = PY-pyruvate

LACT = PY-lactate

PYG + PROP = PYG-propionate

Strains:

0416 = neotype stra1n, Prévot DO-4

0573 = U.S. isolate

1691 - Prévot 1218

(labeled R. pleuriticum)
Products:

3 = propanol

4 = butanol

5 - pentanol

6 - hexanol

$A=\operatorname{acet} 1 c$ actd

$P=$ proplonic ac1d

$B=$ butyric acid

V - valeric acid

$C$ - caprolc acid

H = heptanolc acid

$C^{\prime}=$ caprylic acid

() - substrate uptake 


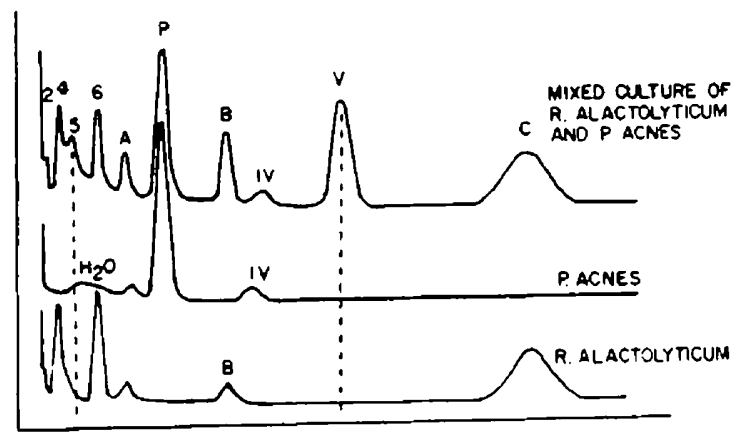

Figure 3. Gas chromatographs of fermentation acids and alcohols of 3-week-old cultures in peptone-yeast extract-glucose medium.

$\begin{array}{llc}2 \text { = ethanol } & \mathrm{A}=\text { acetic acid } & \text { IV = isovaleric } \\ 4=\text { butanol } & \mathrm{P}=\text { propionic acid } & \text { acid } \\ 5=\text { pentanol } & \mathrm{B}=\text { butyric acid } & \\ 6=\text { hexanol } & \text { C = caprioc acid } & V=\text { valeric acid }\end{array}$

P. acnes: see Moore and Cato (1963) and C. acnes, Bergey's Manual, 7th ed. (1957). 
of formic, succinic, or lactic acids and occasionally, traces of butyric acid from fermentation of glucose (Moore and Cato 1963, and unpublished data).

Of 55 sets of analyses on the 33 strains of $\underline{R}$. alactolyticum, none produced catalase, none reduced nitrate, $98 \%$ produced gas in glucose agar deep cultures, none produced indol or fermented glycerol; although some produced weak acid in esculin, none hydrolyzed esculin. We have not observed this particular set of characteristics in any of the 37 strains of catalase-negative anaerobic corynebacteria that we have examined. Therefore, this se $t$ of characteris tics is helpful in differentiating between these two morphologically similar groups. However, analysis of fermentation products from glucose is, at this time, required for certain identification of $R$. alactolyticum. Additional characteristics of $R$. alactolyticum and the degree of variation that may be expected to occur among various strains of the species are presented in Table 1.

\section{FERMENTATION CHARACTERISTICS}

Other species of bacteria that produce caprylic acid are unknown to us. The even-carbon products appear to be synthesized by condensation of a 2-carbon intermediate with carboxylic acids in the medium. When strains of $\underline{R}$. alactolyticum were grown in glucose broth containing sodium propionate, valeric and heptanoic acids and amyl alcohol (pentanol) were produced in addition to the even-carbon acids and alcohols normally formed from fermentation of glucose (Fig. 2). Similarly, amyl alcohol and valeric acidwere found in glucose-broth cultures inoculated with a mixture of $\underline{P}$. acnes and $R$. alactolyticum. Neither organism, grown in pure culture in glucose broth, formed either of these products (Fig. 3).

\section{ACKNOW LEDGMENTS}

The authors wish to acknowledge the assistance of James A. Nash and Lura E. Whiter of the V.P.I. Computing Center; the assistance of Dr. W.B. Gross for photographic reproductions; and the technical assistance of Ann C. Mitchell, Joy W. Skaar, M. Lorna Pais, Susan S. Jennings, Virginia D. Long, Noralea W. Dalkin, Evelyn W. Lindamood, Patricia W. Barber, Lynn M. Napier, and Madge S. Fisher. 


\section{LITERATURE CITED}

Breed, R.S., E.G.D. Murray, and N.R. Smith. 1957. Bergey's Manual of Determinative Bacteriology, 7 th ed. The Williams and Wilkins Co., Baltimore.

Moore, W.E.C. 1966. Techniques for routine culture of fastidious anaerobes. Int. J. Syst. Bacteriol. 16:173190.

and Elizabeth P. Cato. 1963. Validity of Propionibacterium acnes (Gilchrist) Douglas and Gunter comb. nov. J. Bacteriol. 85:870-874; also see erratum, 1965, J. Bacteriol. 89:549. and - 1965. Synonymy of Eubacterium limosum and Butyribacterium rettgeri: Butyribacterium limosum comb. nov. Int. Bull. Bacteriol. Nomen. and Taxon. 15:69-80.

and Lillian V. Holdeman. 1966. Fermentation patterns of some Clostridium species. Int. J. Syst. Bacteriol. 16:383-415.

Prévot, A. R. 1957. Manuel de classification et de détermination des bactéries anaérobies. Masson et cie., Paris.

- 1966. Manual for the classification and determination of the anaerobic bacteria. (translated by V. Fredette) Lea and Febiger, Philadelphia. - M. Raynaud, and M. Digeon. 1947. Sur une espèce anaérobie: Ramibacterium pleuriticum. Ann. Inst. Pasteur 73:481-483.

and J. Taffanel. 1942. Recherches sur une nouvelle espece anaérobie Ramibacterium alactolyticum (nov. spec.). Ann. Inst. Pasteur 68:259-262.

Vinzent, R. and V. Reynes. 1947. Étude d'un nouvel anaérobie: Ramibacterium dentium. Ann. Inst. Pasteur 73:594-595. 